\title{
A DISTRIBUTIONAL HARDY TRANSFORMATION
}

\author{
R.S. PATHAK \\ Department of Mathematics \\ Banaras Hindu University \\ Varanasi, India \\ J.N. PANDEY \\ Department of Mathematics \\ Carleton University \\ ottawa, Canada \\ (Received January 23, 1979)
}

ABSTRACT: The Hardy's F-transform

$$
F(t)=\int_{0}^{\infty} F(t y) \text { yf }(y) d y
$$

is extended to distributions. The corresponding inversion formula

$$
f(x)=\int_{0}^{\infty} c_{\nu}(t x) t F(t) d t
$$

is shown to be valid in the weak distributional sense. This is accomplished by transferring the inversion formula onto the testing function space for the generalized functions under consideration and then showing that the limiting 
process in the resulting formula converges with respect to the topology of the testing function space.

KEY WORDS AND PHRASES. Integral Transform, Hardy Transform, Hankel Transform, Distributions, Generalized Functions.

AMS (MOS) SUBJECT CLASSIFICATION (1970) CODES. Primary 44A20, secondary 46 F05.

\section{INTRODUCTION.}

The Hardy transforms with their inversion formulae are represented by the following two integral equations:

and

$$
f(x)=\int_{0}^{\infty} F_{\nu}(t x) t d t \int_{0}^{\infty} c_{\nu}(t y) y f(y) d y
$$

where

$$
f(x)=\int_{0}^{\infty} c_{\nu}(t x) t d t \int_{0}^{\infty} F_{\nu}(t y) y f(y) d y
$$

$$
C_{\nu}(z)=\operatorname{cosp} \pi J_{\nu}(z)+\operatorname{sinp} \pi Y_{\nu}(z)
$$

and

$$
\begin{aligned}
F_{\nu}(z) & =\sum_{m=0}^{\infty} \frac{(-1)^{m}(z / 2)^{\nu+2 p+2 m}}{\Gamma(p+m+1) \Gamma(p+m+\nu+1)} \\
& =2^{2-\nu-2 p s} \nu+2 p-1, \quad v^{(z)} /\{\Gamma(p) \Gamma(\nu+p)\}[2, p \cdot 40]
\end{aligned}
$$

The theory of the inversion formulae (1) and (2) has been given by Cooke [1] The Hankel transform with its inversion formula can be deduced as a special case of both (1) and (2) by taking $p=0$. The $Y$-transform [3, $p$. 93] is a special case of (1) whereas H-transform [3, p. 155] is a special case of (2) for $p=\frac{1}{2}$. Recently the inversion formula (1) was proved to be valid for the generalized function space $H_{\alpha}^{\prime}$ (I) by Pathak and Pandey [7] in the weak distributional sense. It turns out that the kernal y $F_{\nu}$ (ty) of $F_{\nu}$-transform does not belong to the space 
$\mathrm{H}_{\alpha}(\mathrm{I})$ and therefore the inversion formula (2) cannot be proved to be valid for the space of distributions directly as a corollary to theorems proved in [7]. We will therefore extend briefly the inversion formula (2) to a generalized function space essentially by following the techniques and results proved in [7].

2. TESTING FUNCTION SPACE $\mathrm{H}_{\alpha, \beta}^{\nu, p}$ (I). For $-\frac{1}{2} \leq \nu \leq \frac{1}{2}$ and real p let $\mathrm{F}_{\nu}(z)$ be the function defined in (4) and let $\alpha$ be a fixed number satisfying $\alpha+\nu+2 p \geq 0$. Assume that $\beta$ is also a fixed number satisfying $\beta \geq \sigma=\max \left(\nu+2 p-2,-\frac{1}{2}\right)$. For each $k=0,1,2, \ldots$ define a positive and continuous function $\xi_{k}(x)$ on $I=\{x ; 0<x<\infty\}$ satisfying

$$
\xi_{k}(x)=\begin{array}{rr}
x^{2 k+\alpha} & 0<x \leq 1 \\
x^{-\beta-2} & x>1
\end{array}
$$

An infinitely differentiable complex-valued function $\emptyset(x)$ defined over I is said to belong to the space $H_{\alpha, \beta}^{\nu, p}$ (I) if

$$
\nu_{k}(\theta) \equiv \sup _{0<x<\infty}\left|\xi_{k}(x) \Delta_{x}^{k}\left(\frac{\varrho(x)}{x}\right)\right|<\infty
$$

for each $k=0,1,2,3, \ldots$ where $\Delta_{x}$ stands for the differentiation operator $\left(D_{x}^{2}+\frac{1}{x} D_{x}-\frac{\nu^{2}}{x^{2}}\right), D_{x}=\frac{d}{d x}$. It can be readily seen that $H_{\alpha, \beta}^{\nu, P}$ (I) is a vector space. The topology over ${ }_{\alpha, \beta}^{\nu, p}$ (I) is generated by the sequence of seminorms $\left(\nu_{k}\right\}_{k=0}^{\infty}[9 ; p \cdot 8]$.

A sequence $\left(\emptyset_{\nu}\right\}$ in this space is said to converge to the element $\emptyset$ if 
$\gamma_{k}\left(\emptyset_{\nu}-g\right) \rightarrow 0$ as $\nu \rightarrow \infty$ for each $k=0,1,2,3, \ldots$ A sequence $\emptyset_{\nu}$ in $\mathrm{H}_{\alpha, \beta}^{\nu, p}$ is said to be a Cauchy sequence if $\gamma_{k}\left(\phi_{m}-\varphi_{n}\right) \rightarrow 0$ as $m, n \rightarrow \infty$ independently of each other. It is a simple exercise to verify that the space $H_{\alpha, \beta}^{\nu, P}$ (I) is sequentially complete and so it is a Fréchet space. Since $D(I) \subset \mathrm{H}_{\alpha, \beta}^{\nu, P}$ (I) and the topology of $D(I)$ is stronger than that induced on $D(I)$ by $H_{\alpha, \beta}^{\nu, p}$ (I), it follows that the restriction of any $f \in \underset{\alpha, \beta}{H^{\nu, p}}$ (I) to $D(I)$ is in $D^{\prime}$ (I). In view of the fact that

$$
\Delta_{x}^{k}\left[F_{\nu}(x t)\right]=(-1)^{k} t^{2 k} F_{\nu}(x t)-P(x, t)
$$

where

$$
P(x, t)=t^{\nu+2 p} \sum_{i=1}^{k} a_{i} x^{\nu+2 p-2 i} t^{2 k-2 i}
$$

$a_{k}$ being certain constants depending on $\nu$ and $p$, and the asymptotic orders $[9, \mathrm{p} .345]$

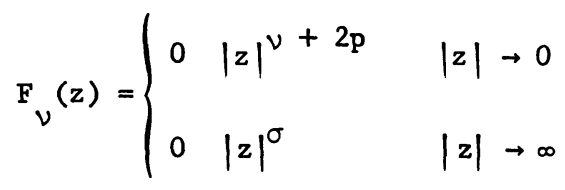

where

$$
\sigma=\max \left(\nu+2 p-2,-\frac{1}{2}\right) \quad[8, \mathrm{pp}, 347,351]
$$

it follows that for fixed $t>0, \quad x F_{\nu}(t x)$ belongs to the space $H_{\alpha, \beta}^{\nu, p}$ (I) when treated as a function of $x$. Therefore, Hardy's $F_{\nu}$-transform $F(y)$ of a generalized function $f \in \underset{\alpha, \beta}{H^{\nu, p}}$ (I) can be defined by

$$
F(y)=\left\langle f(x), \quad x F_{\nu}(x y)\right\rangle, \quad y>0 .
$$

By following the technique as used in [7] it can be shown that $F(y)$ is differentiable for each $\mathrm{y}>0$ and that

$$
F^{\prime}(y)=\left\langle f(x), \frac{\partial}{\partial y}\left\{x F_{\nu}(x y)\right\}\right\rangle
$$


Note that

$$
\frac{\partial}{\partial y}\left[x F_{\nu}(x y)\right] \text { also belongs to } \mathrm{H}_{\alpha, \beta}^{\nu, p} \text { (I). }
$$

We now state some results which will be used in the sequel.

Define

$$
\begin{aligned}
H_{N}(t, x) & =\int_{0}^{N} c_{\nu}(t x) c_{\nu}(x y) y d y \\
& =\frac{N}{x^{2}-t^{2}}\left[x c_{\nu+1}(x N) c_{\nu}(t N)-t c_{\nu+1}(t N) C_{\nu}(x N)\right]-Q(x, t)
\end{aligned}
$$

where

$$
\begin{aligned}
& Q(x, t)=\frac{2 \sin p \pi}{\pi \sin \nu \pi} \sin (p+\nu) \pi \frac{2 \nu 2 \nu}{x^{\nu} t^{\nu}\left(x^{2}-t^{2}\right)}, x \neq t \\
&=\frac{2 \sin p \pi \sin (p+\nu) \pi}{\pi \sin \nu \pi} \frac{\nu}{x^{2}} \quad \text { when } t=x \\
& {[8, p \cdot 466] . }
\end{aligned}
$$

Using the technique employed in proving Lemma 2 in [7] it can be proved that for fixed $t, x \cdot Q(x, t) \in H_{\alpha, \beta}^{\nu, p}(I)$.

It is now a simple exercise to prove for $\alpha \geq|\nu|, \beta \leq \nu-4$ and $\phi \in \mathrm{D}$ (I) that $\mathbf{x} \int_{a}^{b} Q(x, y) \phi(y) y d y$ also belongs to $H_{\alpha, \beta}^{\nu, p}$ (I).

LEMMA 2. Let $\xi_{k}(t)$ be defined as in section 2. Then for $0<y<1$ $\sup _{0<t<\infty}\left|\frac{\xi_{k}(t)}{\xi_{k}(t y)}\right|=y^{\min (\beta+2,-\alpha-2 k}, k=0,1,2, \ldots$

PROOF. The result follows by dividing the $t$ - 1 ine into three parts $0<\mathrm{t}<1, \quad 1<\mathrm{t}<1 / \mathrm{y}, \quad 1 / \mathrm{y}<\mathrm{t}<\infty$ and considering the corresponding

$$
0<t<\infty\left|\frac{\xi_{k}(t)}{\xi_{k}(t y)}\right|
$$


LEMMA 3. Let $c_{\nu}(z)$ be the function as defined in (3) and let $-\frac{1}{2} \leq \nu \leq \frac{1}{2}, \quad \nu-2 \mathrm{p} \leq \alpha \leq 3 / 2, \quad \beta \geq \max \left(\nu+2 \mathrm{p}-2,-\frac{1}{2}\right)$.

Then for fixed $x>0$,

$t \int_{0}^{\eta} F_{\nu}(t y) c_{\nu}$ (xy) ydy $\rightarrow 0$ in $\underset{\alpha, \beta}{H^{\nu, p}}$ (I) as $\eta \rightarrow 0+$

PROOF. The lemma can be proved by using lemma 2 and a variation of the technique used in proving lemma 4 of [7].

LEMMA 4. Let $\alpha, \beta, \nu$ and $p$ be restricted as in Lemma 3 and let f $\in \underset{\alpha, \beta}{H^{\nu, p}}$, then

$$
\int_{0}^{N}\left\langle f(t), t F_{\nu}(t y)>c_{\nu}(x y) y d y=<f(t), t \int_{0}^{N} F_{\nu}(t y) c_{\nu}(x y) y d y>\right.\text {. }
$$

PROOF. The result follows in view of Lemma 3. The details of the technique to be used can be found in [7, Lemma 5].

LEMMA 5. Let $b>a>0$ and $H_{N}(t, x), Q(t, x)$ be the functions as defined by (9) and (10). Then

$$
\lim _{x \rightarrow \infty} \int_{a}^{b}\left[H_{N}(t, x)+Q(t, x)\right] x d x=\begin{array}{lll}
1 & t \in[a, b] \\
0 & t d[a, b]
\end{array}
$$

PROOF. See Lemma 6 in [7].

LEMMA 6. Let the support of $\varphi \in D(I)$ be contained in $(a, b)$ where $b>a>0$. Let $H_{N}(t, x), Q(t, x)$ be the functions as defined in $(9)$ and $(10)$. Assume that $-\frac{1}{2} \leq \nu \leq \frac{1}{2}, \max (-\nu-2 p, \nu) \leq \alpha \leq 3 / 2$ and $\beta \geq \max \left(\nu+2 p-2,-\frac{1}{2}\right)$. Then $t \int_{a}^{b}\left[H_{N}(t, x)+Q(x, t)\right] \emptyset(x) x d x \rightarrow t \emptyset(t) \underset{\alpha, \beta}{\text { in } H_{\alpha, P}^{\nu}}$ (I) $\underline{\text { as } N} \rightarrow \infty$. 
PROOF. The proof can be given only by using Lemma 3 and a simple variations of the techniques used in proving [7, Lemma 7] and so the details are omitted.

\section{INVERSION OF THE DISTRIBUTIONAL}

$F_{\nu}$ - transform: We now state and prove our main result.

THEOREM. Let $-\frac{1}{2} \leq \nu \leq \frac{1}{2}, \max (-\nu-2 \mathrm{p},|\nu|) \leq \alpha \leq 3 / 2$ and $\beta \geq \max$ $\left(\nu+2 p-2,-\frac{1}{2}\right)$

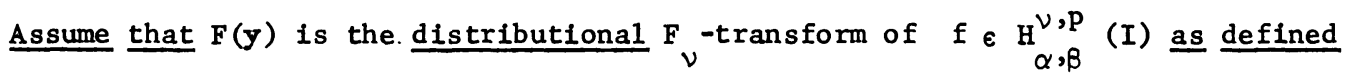
by (7). Then

$$
\lim _{N \rightarrow \infty}\left\langle\int_{0}^{N} F(y) c_{\nu}(x, y) y d y, \phi(x)\right\rangle=\langle f, \phi\rangle \text { for each } \emptyset \in D(I) \text {. }
$$

PROOF. Let the support of $\boldsymbol{g}$ be $[a, b]$ where $b>a>0$.

Since $F(y) C_{V}(x y)$ y generates a regular distribution we have $\int_{0}^{N}\left\langle F(y) c_{\nu}(x y) y d y, g(x)\right\rangle=\int_{a}^{b} \varphi(x) d x \int_{0}^{N} F(y) c_{\nu}(x y) y d y$

$=\int_{a}^{b}\left\langle f(t), \quad t \int_{0}^{N} F_{\nu}(t y) c_{\nu}(x y) y d y\right\rangle \phi(x) d x$

[ Lemma 4]

$=\int_{a}^{b}\left\langle f(t), t\left\{H_{N}(t, x)+Q_{N}(t, x)\right\}\right\rangle g(x) d x$

$=\int_{a}^{b}\left\langle f(t), t\left\{H_{N}(t, x)+Q(t, x)\right\}\right\rangle \phi(x) d x$

for $\mathrm{N}>\mathrm{b}>0$

[1, Lemma $\in \mathrm{p} .394]$ 


$$
=\left\langle f(t), \quad t \int_{a}^{b}\left\{H_{N}(t, x)+Q(t, x)\right\}\right\rangle \frac{Q(x)}{x} x d x
$$

by Riemann Sums technique

$$
[9, \mathrm{p} \cdot 148]
$$

$$
\rightarrow\left\langle f(t), \quad \text { t. } \frac{g(t)}{t}\right\rangle=\langle f, \phi\rangle
$$

This completes the proof of the theorem.

Taking $\mathrm{p}=0$ and $\mathrm{p}=\frac{1}{2}$ in the above theorem we derive COROLLARY 1. Let $f \in \mathrm{H}_{\alpha, \beta}^{\nu, 0}$ (I) where $-\frac{1}{2} \leq \nu \leq \frac{1}{2}, \quad|\nu| \leq \alpha \leq 3 / 2$ and $\beta \geq-\frac{1}{2}$. Define the distributional Hankel transform of $f$ by

$$
F(y)=\left\langle f(t), \quad t J_{\nu}(t y)\right\rangle
$$

then

$$
\lim _{N \rightarrow \infty}\left\langle\int_{0}^{N} F(y) \text { y } J_{\nu}(x y) d y, g(x)\right\rangle=\langle f, \emptyset\rangle
$$

for all $\emptyset_{\varepsilon} D(I)$.

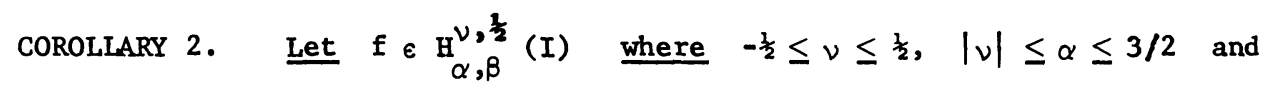
$\beta \geq-\frac{1}{2}$. Define the distributional Struve transform $\left(H_{\nu}\right.$-transform) of $f$ by

$$
F(y)=\left\langle f(t), \quad t \quad H_{\nu}(t y)\right\rangle
$$

then

$$
\lim _{N \rightarrow \infty}\left\langle\int_{0}^{N} F(y) Y_{\nu}(x y) y d y, q(x)\right\rangle=\langle f, \emptyset\rangle
$$

for al1 $\emptyset \in \mathrm{D}(I)$. 
ACKNOWLEDGMENT. This work was supported by National Research Counc11 Grant No. A5298. The first author is thankful to the Banaras Hindu University for granting him study leave.

\section{REFERENCES}

1. Cooke, R.G. The Inversion formulae of Hardy and Titchmarsh, Proc. London Math. Soc. 24 (1925) $381-420$.

2. Erdelyi, A. (Editor) Higher Transcendental Functions, Vol. II, McGraw-Hi11 Book Co., Inc., New York, 1953.

3. Erdelyi, A. (Editor) Tables of Integral Transforms, Vo1. II, McGraw-Hi11 Book Co., Inc., New York, 1954.

4. Hardy, G.H. Some formulae in the theory of Bessel functions, Proc. London Math. Soc. (2), 23, (1925) 1xi-1xiii.

5. Pandey, J.N. and Zemanian, A.H. Complex inversion for the generalized convolution transformation, Pacific J. Math. 25, (1968) 147-157.

6. Pandey, J.N. An extension of Haimo's form of Hankel convolutions, Pacific J. Math. 28 (1969) 641-651.

7. Pathak, R.S. and Pandey, J.N. A distributional Hardy transformation, Proc. Camb. Phil. Soc. 76 (1974) 247-262.

8. Watson, G.N. A treatise on the theory of Bessel functions, Cambridge University Press, 2nd edition, 1962 .

9. Zemanian, A.H. Generalized integral transformations, Interscience Publishers, 1968. 


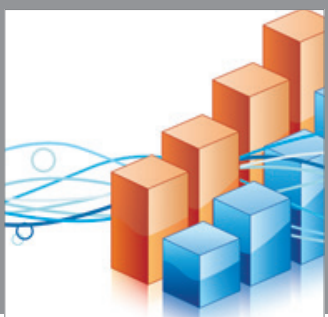

Advances in

Operations Research

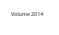

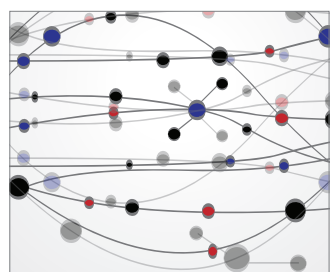

\section{The Scientific} World Journal
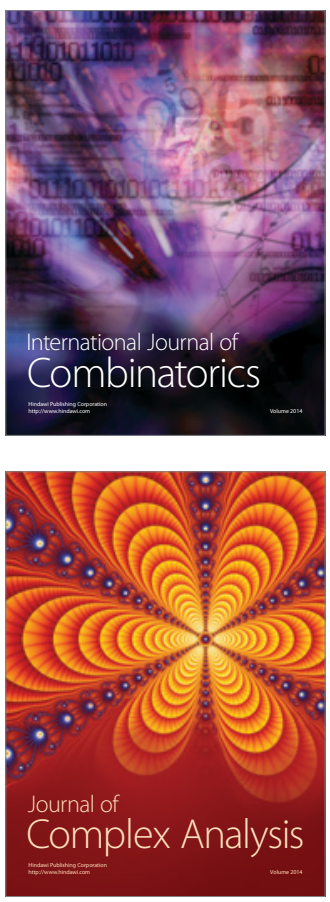

International Journal of

Mathematics and

Mathematical

Sciences
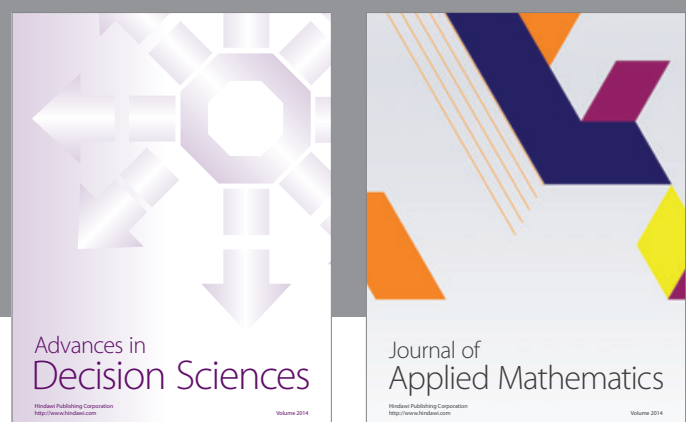

Journal of

Applied Mathematics
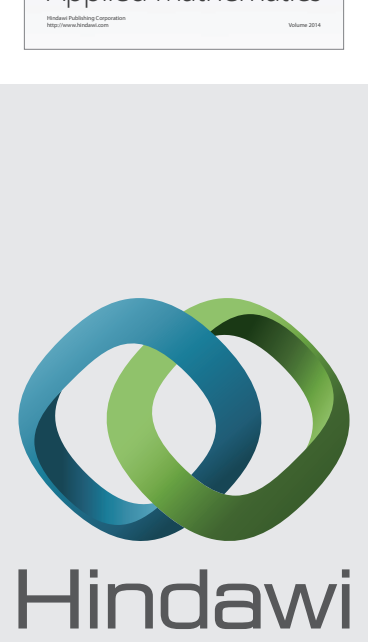

Submit your manuscripts at http://www.hindawi.com
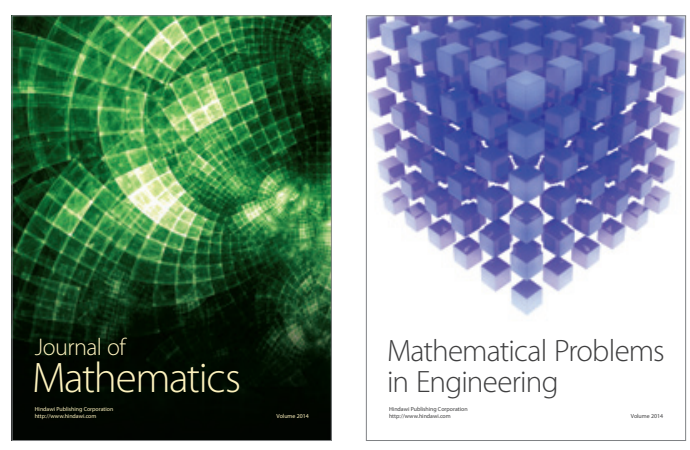

Mathematical Problems in Engineering
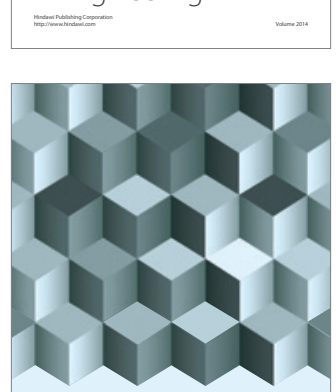

Journal of

Function Spaces
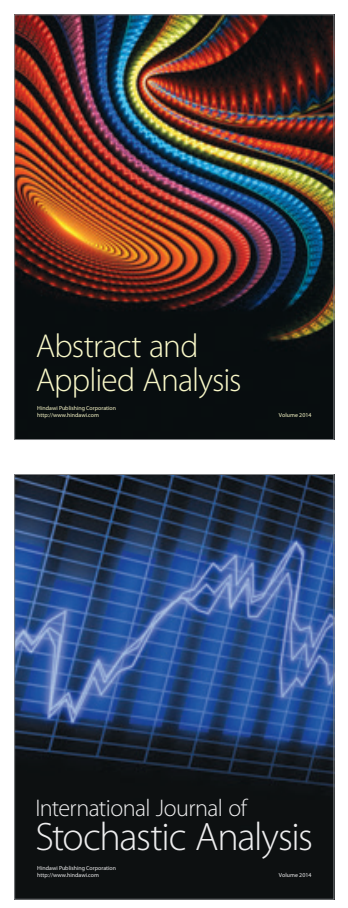

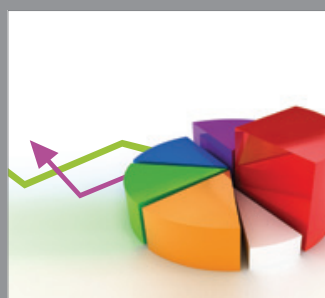

ournal of

Probability and Statistics

Promensencen
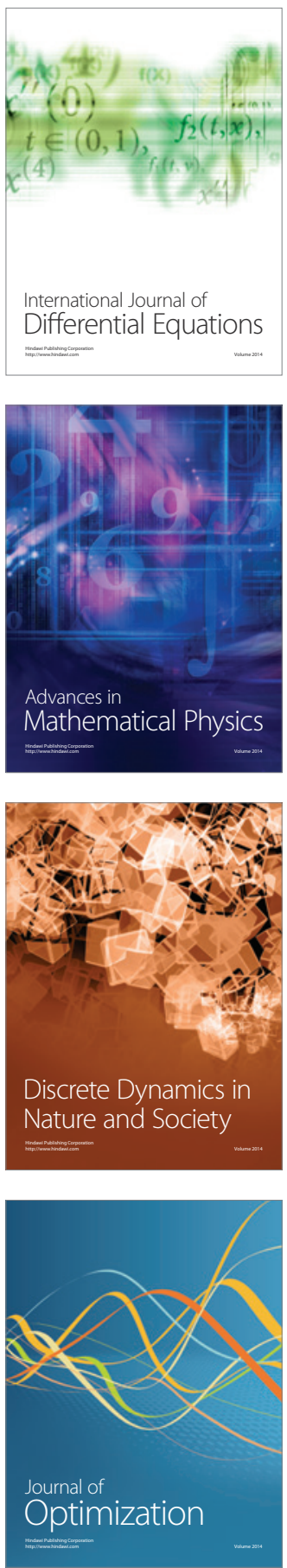